\title{
Representation of multi-connected system of Fuzzy State Space Modeling (FSSM) in potential method based on a network context
}

\author{
Jibril Aminu, Tahir Ahmad ${ }^{*}$ and Surajo Sulaiman \\ Department of Mathematical Science, Faculty of Science, Universiti Teknologi Malaysia, 81310 UTM Johor Bahru, Johor, Malaysia \\ * Corresponding author: tahir@ibnusina.utm.my
}

\section{Article history}

Received 30 January 2017

Accepted 6 December 2017

\begin{abstract}
Every efficient model explains the concept and the representation of the system, the most obvious choice for this mathematical model in a lot of cases is the state space representation. A multiconnected system of fuzzy state space Model is made up of several components, each of which performs a function. These components are interconnected in some manner and determine how the overall system operates. Its the complexity of this system (FSSM) that leads to the main objective of this research. In this paper, we study the concept of graph, network system and network projections which are the requisite knowledge to potential method. Finally, the multi-connected system of FSSM of type A namely feeder, common feeder and greatest common feeder are transformed into potential method using various method of transformation.
\end{abstract}

Keywords: FSSM, potential method, feeder, common feeder, greatest common feeder

\section{INTRODUCTION}

L. A. Zadeh in the early 1960 s proposed a fuzzy set as the general model of uncertainties inherent in engineering and systems (Zadeh, 1965). In his passionate effort, traditional concept was applied in justifying the truth value of membership and true values, respectively. A system is a combination of variable components connected with each other and work together to perform a function (Paraskevopoulos, 2002). A system model is a model that explains the concept and representation of the system. System Modeling plays a vital role in different areas of Engineering (Ismail, 2005). The significant feature of modeling a system is the development of an input and output scheme that will describe behaviour of the system on the working range.

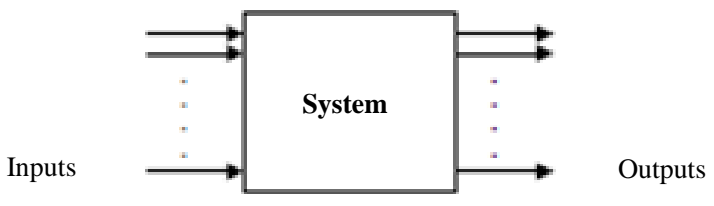

Fig. 1 Showing the mathematical model of a system

Recently, Researches proposed several types of mathematical models for the description of systems (Taufiq, 2007). The selection and the identification of appropriate mathematical representations are of central importance in the analysis of systems. The most obvious choice for this mathematical model in a lot of cases is the state space representation. The state space models came from the state-space equations. However, state-space equation is a description in the time domain which may be applied to a very wide category of systems. The development of the algorithm for FSSM is based on three phases of a fuzzy system; fuzzification, fuzzy environment (Ahmad, 1998) and defuzzification. After which Ismail (2005) developed fuzzy state space modeling (FSSM)

This research is an extension to the research on some aspects of number theory approach on the multi-connect systems of fuzzy state space model in Taufiq (2007). In his work, he explores the concept of number theory to describe connections between systems, in particular interconnected systems of fuzzy state space model (FSSM).

Our aim in this study is to presents multi-connected system of fuzzy state space model in potential method using network projection as discussed by Pedron et al (2011) and Opsahl (2013).

\section{FUZZY STATE SPACE MODELING}

\section{Definition 1}

A Fuzzy State Space Model of a multivariable dynamic system is defined as

$$
\begin{gathered}
S_{g F}: \dot{x}(t)=A x(t)+B \bar{u}(t) \\
\tilde{y}(t)=C x(t)
\end{gathered}
$$

where $\tilde{v}(t)$ denotes the fuzzified input vector $\left[u_{1}, u_{2}, \ldots, u_{n}\right]^{T}$ and $\tilde{y}(t)$ denotes the fuzzified output vector $\left[y_{1}, y_{2}, \ldots, y_{m}\right]^{T}$ with initial conditions as $t_{0}=0$ and $x_{0}=x\left(t_{0}\right)=0$. The elements of state matrix $A_{p X p}$, input matrix $B_{p X n}$, and output matrix $C_{m X p}$ are known to a specified accuracy.

The representation of large-scale systems with the interconnection of many subsystems refers to as fuzzy state space modeling in multiconnected systems(Ismail, 2005). Multi-connected system can be classified based on the source of input parameters; either the input 
parameters are directly from one system (connection of type A) or from multiple systems (connection of type B). The two types of multiconnected systems are described by the following definitions.

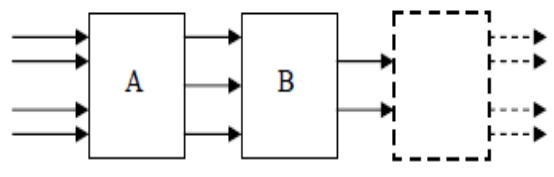

Input variables

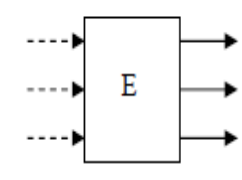

Output variables
Fig. Error! No text of specified style in document. Shows a simple multi-connected system

\section{Definition 2}

Let $S_{g F}$ be a system in the fuzzy state space form specified in (Definition 1). A multi-connected system of $S_{g F}$ is defined as a sequence $S_{g F 1}, S_{g F 2}, \ldots, S_{g F n}(i=1,2, \ldots, n)$ where the output of $S_{g F i}$ are the input of $S_{g F(i+1)}$.

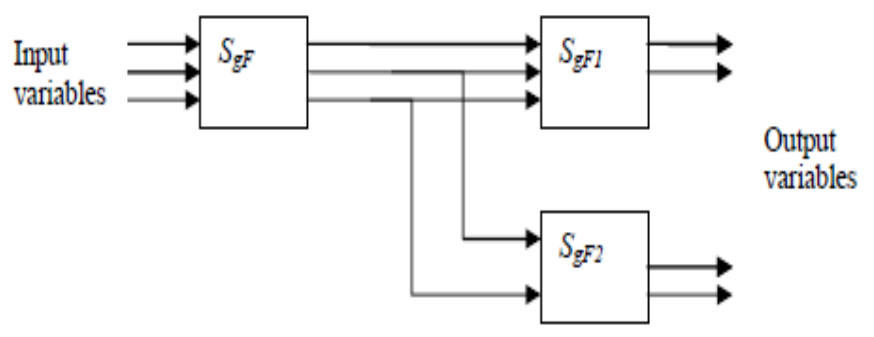

Fig. 3 Multi-connected systems of type A

\section{Definition 3}

Let $S_{g F}$ be a system in the fuzzy state space form specified in (Definition 1). A multi-connected system of $S_{g F}$ is defined as a sequence $S_{g F 1}, S_{g F 2}, \ldots, S_{g F n}$ where the inputs of $S_{g F c}$ come from the combination of other systems, $S_{g F i}$ for $i=1,2, \ldots, n$.

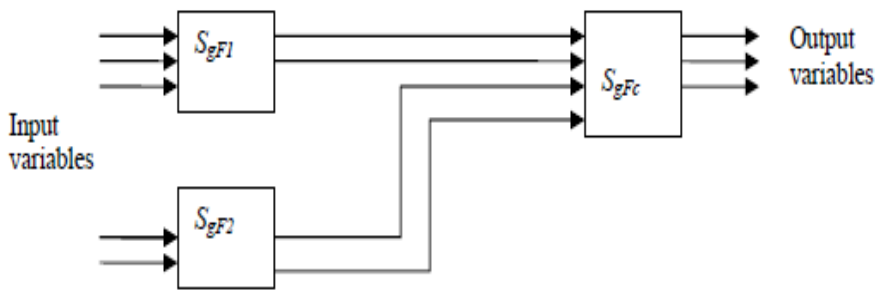

Fig. 4 Multi-connected systems of type B

Algebraic properties of systems of fuzzy state space model (FSSM) was first developed by Ismail (2005), the objective of that study is to provide an insight for further exploration of the algebraic structures for systems of FSSM. She further discussed on the algebraic view of multiconnected systems of FSSM which was deduced from the fundamental concepts on number theory. The basic concepts of divisor, common divisor and greatest common divisor from properties of the integers are adapted to give a deeper understanding and some physical interpretation for a system of FSSM.

Let $S_{g F 1}, S_{g F 2}, \ldots S_{g F n}(i=1,2, \ldots, n)$ be sequence of systems of Fuzzy State Space Model (FSSM)c such that

\section{Definition 4}

For $S_{g F 1}, S_{g F 2} \in S_{G F}, S_{g F 1}$ is a feeder of $S_{g F 2}$ (written as $\left.S_{g F 1} \mid S_{g F 2}\right)$ if and only if any output of $S_{g F 1}$ is/are the inputs of $S_{g F 2}$.

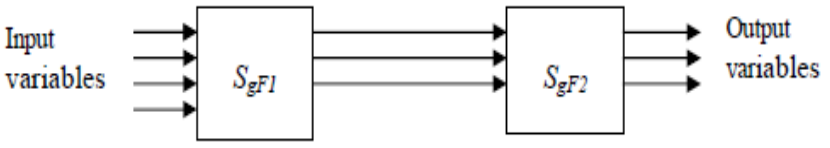

Fig. 5 Showing how $S_{g F 1}$ feed $S_{g F 2}$

\section{Definition 5}

For $S_{g F 1}, S_{g F 2} \in S_{G F}, S_{g F}$ is called a common feeder of $S_{g F 1}$ and $S_{g F 2}$ if $S_{g F} \mid S_{g F 1}$ and $S_{g F} \mid S_{g F 2}$.

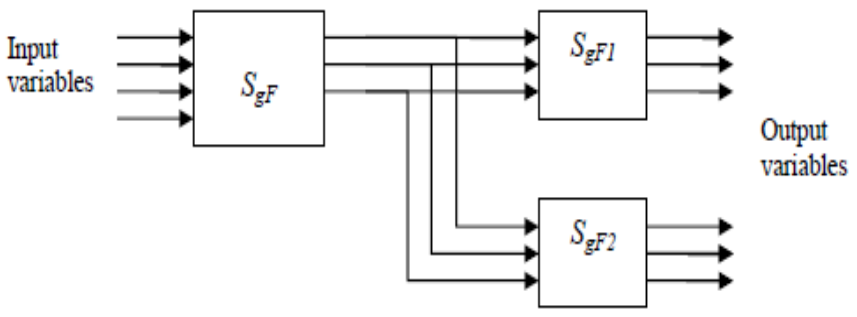

Fig. 6 Showing how $S_{g F}$ feeds of $S_{g F 1}$ and $S_{g F 2}$

\section{Definition 6}

For $S_{g F 1}, S_{g F 2} \in S_{G F}, S_{F F}$ is called the greatest common feeder of $S_{g F 1}$ and $S_{g F 2}$ written as $g c f\left(S_{g F 1}, S_{g F 2}\right)$ if

(i) $\quad S_{g F} \mid S_{g F 1}$ and $S_{g F} \mid S_{g F 2}$.

(ii) $\quad S_{F F} \mid S_{g F 1}$ and $S_{F F}\left|S_{g F 2} \rightarrow S_{F F}\right| S_{g F}$.

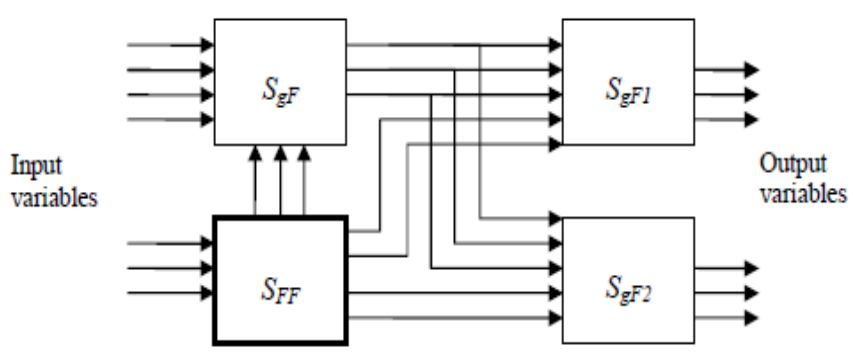

Fig. $7 S_{F F}$ is the greatest common feeder of $S_{g F 1}$ and $S_{g F 2}$

This term "feeder" is used in a system of FSSM to denote the source of the input parameters of the system. In this case, the idea of divisor is used to represent a simple interconnection system.

\section{POTENTIAL METHOD}

The concept of potential method was first developed by (Caklovic, 2002), in his studies 'decision making via potential method' and was originally created for subjective decision making. The PM uses a preference graph to capture the results of pair wise comparisons. However, (Caklovic, 2003) gives the brief description of potential method in order to introduce a dissimilarity measure on a certain class of oriented graphs arising in the context of group decision making. 


\section{Definition 7}

Suppose $n$ and $m$ are denoted as the cardinality of $V$ and $\mathcal{A}$ respectively. Incidence matrix of the preference graph is denoted by $B$ and it is $m \times n$ matrix defined by

$$
B_{e, v}=\left\{\begin{array}{c}
-1, \text { if eleaves } v \\
1, \text { if e enters } v \\
0, \text { otherwise }
\end{array}\right.
$$

Let $F$ be a given preference flow, and $B$ incidence matrix of the graph. Let us assume, for simplicity, that the graph is weakly connected The system

$$
B^{T} B X=B^{T} F, \sum_{i=1}^{m} X_{i}=0
$$

Eq (1) is one-dimensional affine space. Each solution of this equation represents 'utility' on the sets of alternatives that should be invariant on adding a constant to each vertex. The unique solution of Eq (1) is called normal integral of $F$. The function $X: V \rightarrow \mathbb{R}$ is called 'potential method'.

\section{Definition 8}

A graph $G=(V, E)$ is a mathematical structure consisting of two finite sets $V$ and $E$. The elements $V$ are called vertices (or nodes) and the elements of $E$ are called edges.

\section{Definition 9}

A directed graph is a graph or a set of objects (called vertices or nodes) that are connected together, where all the edges are directed from one vertex to another. A directed graph is sometimes called a digraph or a directed network.

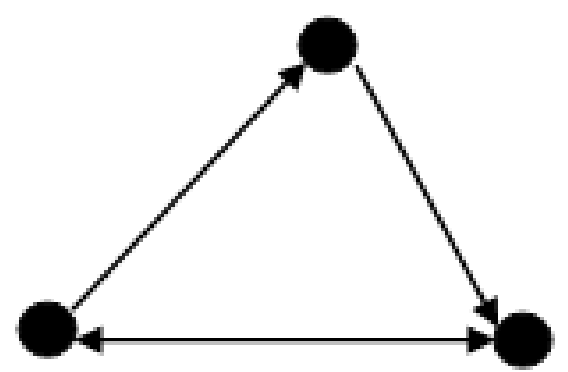

Fig 8 A simple directed graph.

\section{Definition 10}

Suppose $V$ is an alternatives set, then a pair $\alpha=(u, v) \in V \mathrm{x}$ $V$ is declared to be an arc of a directed graph if $v$ is more preferred than $u$ with weight of that preference a non-negative number. The set of all arcs is donated by $\mathcal{A}$

\section{Definition 11}

A function $F: \mathcal{A} \rightarrow \mathbb{R}$ which assigns to each $\operatorname{arc} \alpha \in \mathcal{A}$ its weight is called a preference flow. The flow component $F(\alpha)$ is usually taken from a given interval of real number.

Preference flow is always non-negative and can be represented as $m \times 1$ matrix over the interval of real number. Oriented graph $(V, \mathcal{A})$ is called preference graph.

\section{Definition 12}

Preference graph is complete if each pair of alternatives is compared i.e. if for each pair $(i, j)$ of vertices $(i, j) \in \mathcal{A}$ or $(j, i) \in \mathcal{A}$. For a given flow $F$ on the preference graph $(V, \mathcal{A})$ and $\alpha \in \mathcal{A}$ we use a convention $F(-\alpha)=-F(\alpha)$.

\section{Definition 13}

The matrix $B_{e, v}=[i, j]$ of the graph $G=(V, E)$ with $V=$ $\left\{v_{1}, v_{2}, \ldots, v_{n}\right\}$ and $E=\left\{e_{1}, e_{2}, \ldots, e_{m}\right\}$, is the $n \times m$ matrix associated with a chosen orientation of the edges of $G$ in which for each $e=\left(v_{i}, v_{j}\right)$, one of $v_{i}$ or $v_{j}$ is taken as positive end and the other as negative end, and is defined by

$$
B_{e, v}=\left\{\begin{array}{c}
-1, \text { if eleaves } v \\
1, \text { if e enters } v \\
0, \text { otherwise. }
\end{array}\right.
$$

\section{Example 1}

We use an example of a directed graph in Figure 6 to develop its incidence matrix. This will help us to get a full insight of how incidence matrix is been developed. Now, we consider a directed graph $G$ with four (4) nodes a, b, c and d together with five (5) edges, which are directed either toward or away from each node.

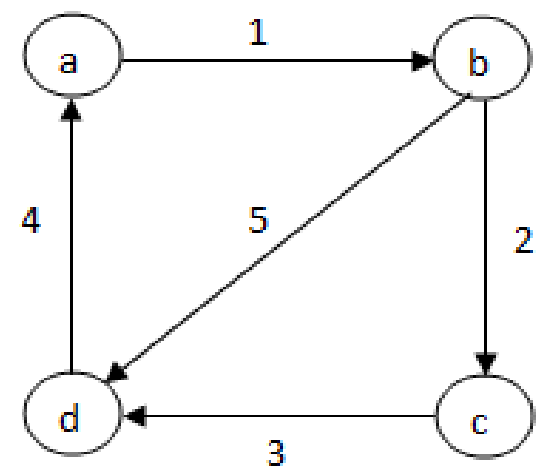

Fig. 9 A simple directed graph G.

The figure below represents the incidence matrix of the above directed graph. The matrix has the entries of $-1,1$ and 0 . If the edge is leaving a node then, the entry is -1 and if it's coming toward a node then, the entry is 1 otherwise the entry is 0 .

$$
B_{\alpha, i}=\left[\begin{array}{ccccc}
-1 & 0 & 0 & 1 & 0 \\
1 & -1 & 0 & 0 & -1 \\
0 & 1 & -1 & 0 & 0 \\
0 & 0 & 1 & -1 & 1
\end{array}\right]
$$

Fig. 10 Incidence matrix B derived from Fig. 9 .

\section{Network system and projection}

A network is a set of items, called vertices or nodes, with connections between them; called edges. Systems taking the form of networks are also called "graphs" in much of the mathematical literatures (Newman, 2003). Many network dataset are by definition two-mode networks known as affiliation or bipartite networks (Opsahl, 2013). These are a particular type of networks with two sets of nodes and ties that are only established between nodes belonging to different sets.

Two-mode networks are rarely analysed without transforming them (Opsahl, 2013). This is because most network measures are solely defined for one-mode networks, and only a few of them have been redefined for two-mode networks. Transforming a two-mode network to a one-mode network is often done using a method known as projection. However, our work will consider the multi-connected system of FSSM and represents it to two-mode network before transforming it to one-mode network in particular potential Method. The diagram in the following figure illustrates an example of FSSM in form of two-mode network where the $S_{g F}{ }^{\prime} s$ represents the node set with ties between the nodes. 


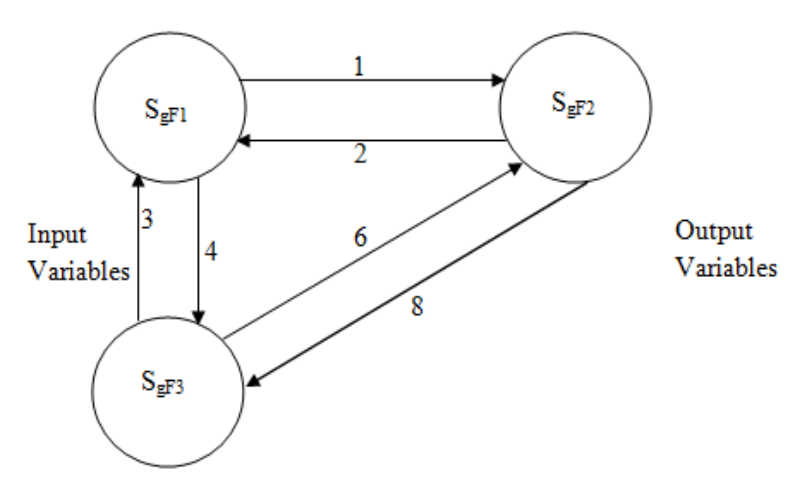

Fig. 11 An example of two-mode network of FSSM

Types of projection method for two-mode network

Here, methods of projection have been discusses to transform the two-mode network into one-mode network; binary, Sum and weighted Newman.

1. In binary method of projection, only connectivity is considered without including the weight of the links. A binary unimodal network is created with cells representing presence of interaction $\left(x_{i j}=1\right.$ when at least one node is connected to the other) otherwise $\left(x_{i j}=0\right.$ when there is no connection between nodes or systems). In case of its weight, it is formalized as

$$
w_{i j}=\sum_{p} 1
$$

where $w_{i j}$ is the weight between the nodes of the system. In the case of our multi-connected system of FSSM, the nodes are either $S_{g F 1}, S_{g F 2}, S_{g F}$ or $S_{g F F}$. However, $p$ represents the number of the nodes that are connected to each other, i.e. their co-occurrences. This method of projection does not include the actual weight of the links after projection.

2. Sum method calculates the weights, but does not divide the co-occurrence of weights; it is formalized as

$$
w_{i j}=\sum_{p} w_{i . p}
$$

where $w_{i . p}$ is the weight of either $S_{g F 1}, S_{g F 2}, S_{g F}$ or $S_{g F F}$ with the co-occurrence as compared to other nodes.

3. Newman (2001) extended the previous procedure while working with scientific collaboration networks. However, based on our context if many edges are connected to the same node, then the bonds among the edges that connect to a particular node is stronger than the bonds connected between more than one nodes. The bonds weakens as the number of edges increasing. It is formalized as

$$
w_{i j}=\sum_{p} \frac{1}{N_{p}-1}
$$

where $w_{i j}$ is the weight between the nodes of the system. $N_{p}$ is the number of edges in between the vertices and $p$ represents the number of the nodes that are connected to each other, i.e. their co-occurrences.

\section{Network and multi-connected Fuzzy State Space Systems}

We have since discussed network systems as very useful in describing connectivity between variety types of system. A set of vertices joined by edges is the simplest type of network. There are many ways in which networks may be more complex than this. For instance, there may be more than one different type of vertex in a network, or more than one different type of edge. Furthermore, vertices or edges may have a variety of properties, numerical or otherwise, associated with them. As earlier stated, we will confine our work base on multiconnected system of FSSM of type $A$. Now, we consider the feeder, common feeder and greatest common feeder illustrated in Figures 12, 13 and 14 as two-mode network systems. Such that taking the $S_{g F 1}$, $S_{g F 2}, S_{g F}$ and $S_{g F F}$ as the vertices and the interconnection between them represent the edges.

Figure 12 shows the two-mode network of feeder with a ties of 3 and 4 between node $S_{g F 1}$ and $S_{g F 2}$. This shown that $S_{g F 1}$ is connected to $S_{g F 2}$.

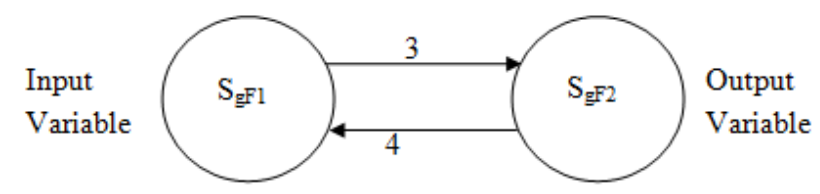

Fig 12 Two-mode network system of a feeder.

Figure 13 shows a two-mode network of a common feeder with a ties of 3 and 2 between $S_{g F}$ and $S_{g F 1}$. A ties of 4 and 3 between $S_{g F}$ and $S_{g F 2}$. This also shows that $S_{g F}$ is connected with $S_{g F 1}$ and $S_{g F}$ is connected with $S_{g F 2}$.

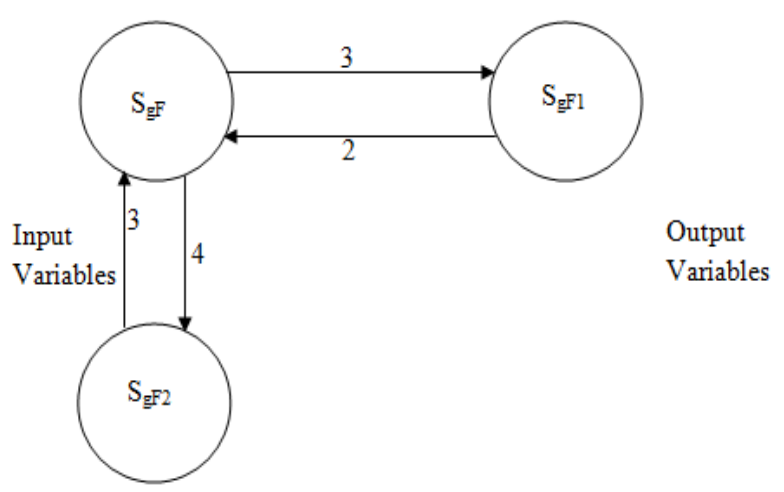

Fig 13 Showing two-mode network of a common feeder.

Figure 14 shows a two-mode network of a greatest common feeder with a ties of 4 and 3 between $S_{g F}$ and $S_{g F 1}, 3$ and 2 between $S_{g F}$ and $S_{F F}$, and 5 and 6 between $S_{F F}$ and $S_{g F 2}$. There is also a ties of 6 and 8 between $S_{F F}$ and $S_{g F 1}$ and a ties of 7 and 5 between $S_{g F}$ and $S_{g F 2}$. This shown that $S_{g F}$ is connected with $S_{g F 1}, S_{g F}$ is connected with $S_{F F}, S_{F F}$ is connected with $S_{g F 2}$. Also $S_{g F}$ is connected with $S_{g F 2}$ and $S_{F F}$ is connected with $S_{g F 1}$.

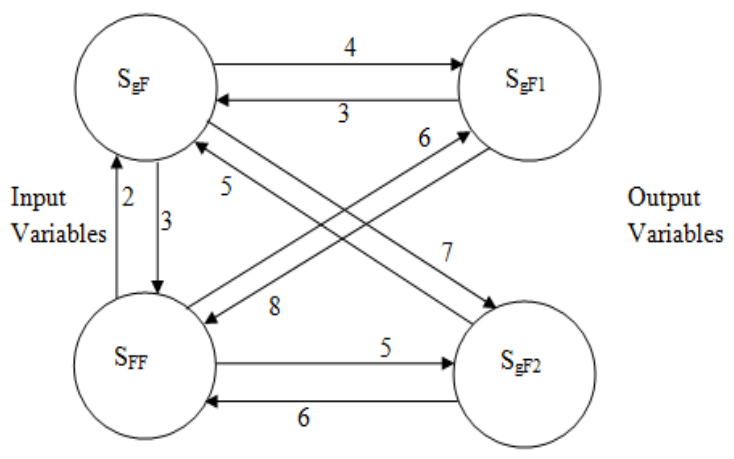

Fig 14 Two-mode network of a greatest common feeder 
Projection is done by selecting one of the sets of nodes and linking two nodes from that set if they were connected to the same node. Now, we need to show the mutuality of the vertices between each of the system which at the same time will show the transformation of the multi-connected system of FSSM to PM form.

We consider the binary, sum and Newman methods of projection in transforming the systems since there is a connection directed or pointing between the vertices.

In Binary method of projection, only connectivity is considered without including the weight of the links. By default, since there is connection between $S_{g F 1}, S_{g F 2}, S_{g F}$ and $S_{g F F}$ the weight remains one (1) otherwise is zero (0).

Now, to compute the sum and Newman method we use the Equations 3 and 4 to find the weight of feeder, common feeder and greatest common feeder as follows:

\section{- $\quad$ Feeder}

1. Sum Method

$$
\begin{aligned}
& w_{i j}=\sum_{p} w_{i . p} \\
& w_{i j}=\sum 3+4=7
\end{aligned}
$$

2. Weighted Newman Method

$$
\begin{aligned}
& w_{i j}=\sum_{p} \frac{1}{N_{p}-1} \\
& w_{i j}=\sum \frac{1}{2-1}=1
\end{aligned}
$$

\section{- Common Feeder}

1. Sum Method

$$
\begin{aligned}
& w_{i j}=\sum_{p} w_{i \cdot p} \\
& w_{i j}=\sum(3+2)+(4+3)=12
\end{aligned}
$$

2. Weighted Newman Method

$$
\begin{aligned}
& w_{i j}=\sum_{p} \frac{1}{N_{p}-1} \\
& w_{i j}=\sum\left(\frac{1}{2-1}\right)+\left(\frac{1}{2-1}\right)=2
\end{aligned}
$$

\section{- Greatest Common Feeder}

1. Sum Method

$$
\begin{aligned}
& w_{i j}=\sum_{p} w_{i \cdot p} \\
& w_{i j}=\sum(4+3)+(3+2)+(5+6)+(7+5)+(6+8)=49
\end{aligned}
$$

2. Weighted Newman Method

$$
w_{i j}=\sum_{p} \frac{1}{N_{p}-1}
$$

$$
w_{i j}=\sum\left(\frac{1}{2-1}\right)+\left(\frac{1}{2-1}\right)+\left(\frac{1}{2-1}\right)+\left(\frac{1}{2-1}\right)+\left(\frac{1}{2-1}\right)=5
$$

Table 1: The weight values of feeder, common feeder and greatest common feeder obtained after projection

\begin{tabular}{lccc}
\hline & $\begin{array}{c}\text { Binary } \\
\text { method }\end{array}$ & $\begin{array}{c}\text { Sum } \\
\text { method }\end{array}$ & $\begin{array}{c}\text { Weighted } \\
\text { Newman }\end{array}$ \\
\hline Feeder & 1 & 7 & 1 \\
Common feeder & 1 & 12 & 2 \\
Greatest common feeder & 1 & 49 & 5 \\
\hline
\end{tabular}

Figure 15 shows the projected network of Feeder projection. As shown in Figure 12, $S_{g F 1}$ was connected with $S_{g F 2}$.

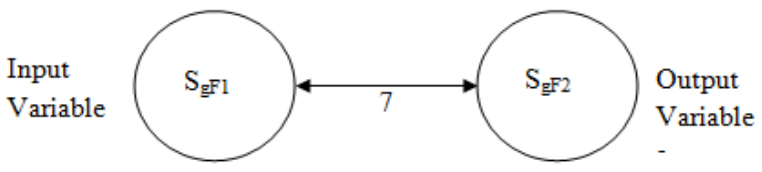

Fig 15 The projected network of feeder after projection.

Figure 16 represents the projected network of common feeder after projection. As shown in Figure 13, $S_{g F}$ was connected with $S_{g F 1}$ and $S_{g F 2}$. So by this co-occurrence, $S_{g F 1}$ is connected with $S_{g F 2}$.

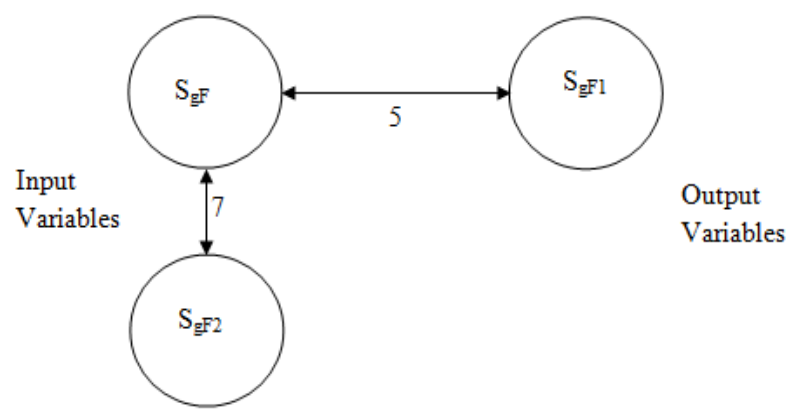

Fig 16 The projected network of common feeder after projection.

Figure 17 represents the projected network of greatest common feeder. As shown in Figure 14, $S_{g F}$ was connected with $S_{g F 1}, S_{g F}$ was connected with $S_{F F}$ and $S_{F F}$ was connected with $S_{g F 2}$. However, $S_{g F}$ was connected with $S_{g F 2}$ and $S_{F F}$ was also connected with $S_{g F 1}$. So by this co-occurrence, $S_{g F 1}$ is connected with $S_{g F 2}$.

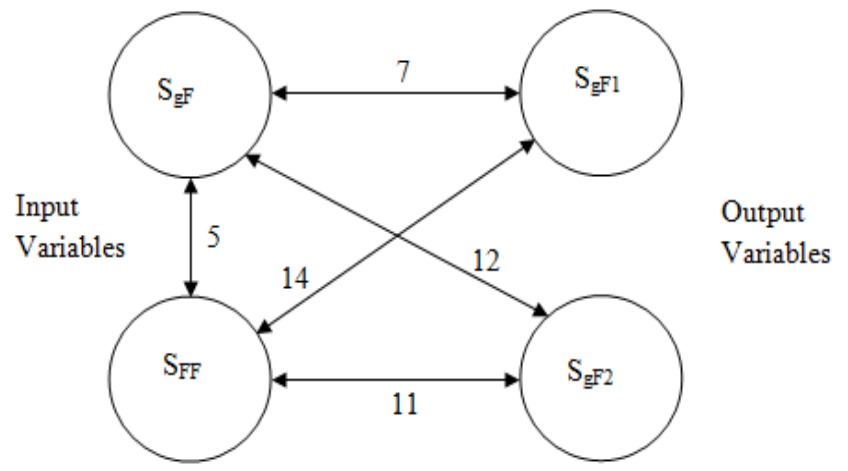

Fig. 17 The projected network of greatest common feeder after projection. 


\section{CONCLUSION}

In conclusion, this research have been able to study the development of fuzzy state space modeling (FSSM). Being it a fundamental concept that so many researchers work with, among others was Ismail (2005) and Taufiq (2007). Indeed, the concept of potential method was also brought to book after we study the requisite knowledge of graph theory and network system. Now, the present research introduces a projection to further study these multi-connected systems. Base on that, we use that projection to show that there is connectivity between systems. To make thing easier, we represent multi-connected system of FSSM into a network system, which easily transform into potential method.

\section{ACKNOWLEDGEMENT}

This work was financially supported by the Universiti Teknologi Malaysia under the research University grant no. FGRS grant 4F756 and Ministry of Higher Education Malaysia.

\section{REFERENCES}

Ahmad, T. (1998). Mathematical and fuzzy modeling of interconnection in integrated circuit. Doctor of Philosophy Thesis, Sheffield Hallam University, Sheffield, United Kingdom.

Caklovic, L. (2002). Decision making by potential method. Preprint.

Caklovic, L. (2003). Graph distance in multicriteria decision making context. Metodološki zvezki, 19, 25-34.

Ismail, R. (2005). Fuzzy state space modeling for solving inverse problems of multi-variable dynamic systems. Doctor of Philosophy Thesis. Universiti Teknologi Malaysia, Johor, Malaysia.

Newman, M. E. J. (2001). Scientific collaboration networks. II. Shortest paths, weighted networks, and centrality. Physical Review E, 64, 016132.

Newman, M. E. J. (2003). The structure and function of complex networks. Society for Industial and Applied Mathematics, 45(2), 167-256

Opsahl, T. (2013). Triadic Closure in two-mode networks: Redefining the global and local clustering coefficients. Social Networks 35, doi: 10.1016/j.socnet.2011.07.001

Paraskevopoulos, P. N. (2002). Modern control engineering. New York: Marcel Dekker.

Padrón, B., Nogales, M., Traveset, A. (2011). Alternative approaches of transforming bimodal into unimodal mutualistic networks. The usefulness of preserving weighted information. Basic and Applied Ecology, 12(8), 713-721.

Taufiq A., K. (2007). Some aspects of number theory approach on the multiconnect systems of fuzzy state space model. Master Dissertation. Universiti Teknologi Malaysia.

Zadeh, L. A. (1965). Fuzzy sets. Information and Control 8.3: 338-353. 